Article

\title{
Quality Monitoring for Laser Welding Based on High-Speed Photography and Support Vector Machine
}

\author{
Teng Wang ${ }^{1, *}$, Juequan Chen ${ }^{1}$, Xiangdong Gao ${ }^{2}$ and Wei $\mathrm{Li}^{3}$ \\ 1 School of Computer Science, South China Normal University, Guangzhou 510631, China; \\ jqchen2015021220@outlook.com \\ 2 School of Electromechanical Engineering, Guangdong University of Technology, Guangzhou 510090, China; \\ gaoxd@gdut.edu.cn \\ 3 School of Physics and Telecommunication Engineering, South China Normal University, \\ Guangzhou 510631, China; liwei@m.scnu.edu.cn \\ * Correspondence: wangteng@m.scnu.edu.cn; Tel.: +86-20-8521-5418
}

Academic Editors: Jiwang Yan and Cem Selcuk

Received: 2 November 2016; Accepted: 10 March 2017; Published: 20 March 2017

\begin{abstract}
In order to improve the prediction ability of welding quality during high-power disk laser welding, a new approach was proposed and applied in the classification of the dynamic features of metal vapor plume. Six features were extracted through the color image processing method. Three features, including the area of plume, number of spatters, and horizontal coordinate of plume centroid, were selected based on the classification accuracy rates and Pearson product-moment correlation coefficients. A support vector machine model was adopted to classify the welding quality status into two categories, good or poor. The results demonstrated that the support vector machine model established according to the selected features had satisfactory prediction and generalization ability. The classification accuracy rate was higher than $90 \%$, and the model could be applied in the prediction of welding quality during high-power disk laser welding.
\end{abstract}

Keywords: laser welding; metal vapor plume; Pearson product-moment correlation coefficient; support vector machines

\section{Introduction}

As an important new laser processing technology, high-power disk laser welding has been increasingly widely used in the manufacturing field. Welding stability monitoring plays an important role in the modern welding process. Therefore, proper sensors and signal processing methods are helpful for detecting the possible presence of weld defects.

Various kinds of sensor methods are applied in the field, such as the use of photodiode signals [1-3], spectroscopy [4,5], high-speed photography [6-8], acoustic signals [9,10], electrical signals [11], X-rays [12], and so on. Among these sensor methods, high-speed photography, which can observe the interaction region between the laser and the workpiece directly, is a kind of non-destructive inspection technique. It has become one of the main development trends for online monitoring of the welding process. Zhang et al. [7] designed an active vision system using a high-speed camera to obtain the morphology information of a molten pool during high-power disk laser welding. The casting shadows of the molten pool were analyzed, and four features, including the area, maximal width, and tilt of casting shadow, as well as maximal distance between casting shadow and the keyhole position, were defined. Pinto-Lopera et al. [13] applied a passive vision system using a high-speed camera and an optical filter in real-time measuring of the width and height of weld beads during gas 
metal arc welding. The use of optical filter and the existence of radiation generated from the electric arc were sufficient to extract features of the images. The measurement process took less than 3 milliseconds per image, which allowed a transfer rate of more than 300 frames per second. Li et al. [14] adopted a high-speed camera and an X-ray transmission imaging system to observe and study the generated spatter and the dynamic molten pool during the high-power laser welding process under different welding parameters. It was found that the ejecting spatter might result in a loss of molten metal and lead to weld defects during laser welding when the focal position was above and on the metal surface.

Machine learning algorithms are commonly used in the signal processing method, which are applied in the design, prediction, monitoring and control of the manufacturing process [14-16]. Shao et al. $[17,18]$ developed new monitoring algorithms respectively based on cross-validation and a quadratic classifier for ultrasonic metal welding of batteries. Wu and Suriano $[19,20]$ applied a classical statistical process control method in manufacturing process monitoring. Among various machine learning algorithms, support vector machine (SVM) is one method widely used in the manufacturing process. It is based on statistical theory and shows great advantage and good development prospects for resolving small sample, nonlinear, and high dimensional pattern recognition problems [21,22]. You et al. [15] applied multisensors to monitor weld defects during high-power disk laser welding. A photodiode sensor was used for monitoring the visible light radiation, an ultraviolet vision sensor was used for plumes and molten pools, and a visual vision sensor was used for monitoring the dynamic behavior of molten pools and keyholes. The SVM classifier has been proven to be efficient in classifying the weld defects in the laser welding process. Mekhalfa et al. [23] studied the use of SVM in the automatic classification of weld defects in radiographic images. The experimental results showed that the SVM classifier was an efficient automatic weld defect classification algorithm. Chen et al. [24] demonstrated that the SVM model was able to predict the Gas Tungsten Arc Welding process correctly. Gao et al. [25] adopted the SVM model to study the relationship between the dynamic characteristics of the arc welding process and welding results. As can be seen, the applications of SVM in the field of welding are quite extensive. However, the computational cost of the SVM algorithm is always very large, due to the strong linear correlations between the input data sets of SVM. Therefore, all the data need to be preprocessed before they are trained.

Real-time monitoring system of laser welding process is the basis for realizing its production automation. The signal of laser-induced plume features can directly reflect the stability of the welding process dynamically, studies conducted on the relationship between the features of metal vapor plume and the quality of welding process in high-power disk laser welding have great significance and application value for the development of real-time monitoring. But it is of highly transient and nonlinearity, which makes it difficult to obtain analytical nonlinear model with accurate quantification. And the information sources are often mixed with lots of noise, which affects the efficiency and accuracy of the extracting feature seriously. One of the key issues that the modeling of real-time monitoring system facing is to resolve how to process and analyze such features better, in order to make it possible to reflect the quality of welding accurately.

In this paper, high-speed photography was used to obtain the transient image sequence of the laser-induced metal vapor during high-power disk laser welding. The dynamic characteristic changes were captured and extracted through a color image processing method. A SVM combined with Pearson product-moment correlation coefficient was used to select the features. Then, a SVM model based on the features was employed to classify the welding quality status in order to provide experimental evidence for the welding prediction and the welding process control.

\section{Experimental Method}

\subsection{Experimental Details}

The experimental scheme shown in Figure 1 was set up. The laser source was a high-power disk laser (TruDisk 10003, TRUMPF, Ditzingen, Germany), with a constant laser power of $10 \mathrm{~kW}$, 
which was used to produce a high-intensity laser beam. The beam diameter of the laser spot was $480 \mu \mathrm{m}$, and the wavelength of the laser was $1030 \mathrm{~nm}$. A MOTOMAN 6-axis robot (EA1400N, Yaskawa, Kitakyushu, Japan) was responsible for controlling the scanning track of the laser beam on the surface of specimen. The welding speed was $4.5 \mathrm{~m} / \mathrm{min}$. The shielding gas was argon in order to ensure the chemical stability of the welding surface environment, and the nozzle angle was $45^{\circ}$. A high-speed camera system (Memrecam fx RX6, NAC, Tokyo, Japan), with complementary metal oxide semiconductor (CMOS) image sensors, was mounted to record the plume images. The frame rate was $2000 \mathrm{f} / \mathrm{s}$, and the image resolution was $512 \times 512$. An optical filter was placed in front of RX6 to filter unnecessary signals. The spectral band-pass filter setup on camera was 320-750 nm (measurement of UV and visible light induced plume and spatter). The specimen was a stainless steel 304 plate with dimensions of $119 \mathrm{~mm} \times 51 \mathrm{~mm} \times 20 \mathrm{~mm}$. The intensity and the direction of the shielding gas remained unchanged during the welding process, and its impact on the state of plume was relatively stable. To simplify the model, the impact of shielding gas was not considered. Other parameters in the laser welding are shown in Table 1.

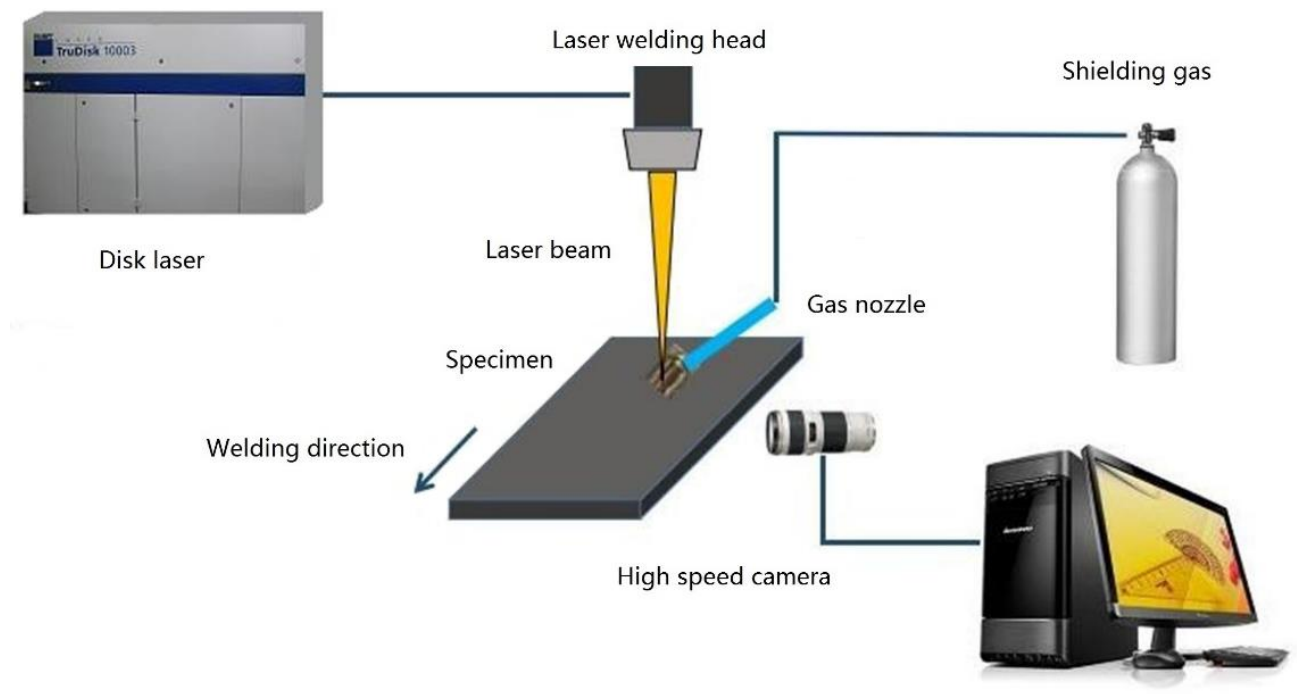

Figure 1. Schematic diagram of the experimental system.

Table 1. Other laser welding conditions.

\begin{tabular}{ccccc}
\hline Beam Quality & Focal Length & Shutter Speed & Pixel Equivalent & Gas Flowing Rate \\
\hline $12 \mathrm{~mm}-\mathrm{mrad}$ & $300 \mathrm{~mm}$ & $0.5 \mathrm{~ms}$ & $11.4 \mathrm{pixel} / 0.1 \mathrm{~mm}$ & $40 \mathrm{~L} / \mathrm{min}$ \\
\hline
\end{tabular}

\subsection{Image Processing}

The image processing was accomplished by using a Matlab platform. The schematic diagrams of color image processing are shown in Figure 2a-e. An original metal vapor image is shown in Figure 2a. In order to reduce the amount of calculations and facilitate subsequent analyses, the original image was cut into $320 \times 320$, and the key region was extracted, as shown in Figure $2 \mathrm{~b}$. Compared to RGB (red, green, blue) color space, the HSI (hue, saturation, intensity) color space was better due to two reasons. First, it uses three components of color image: hue $(H)$, saturation $(S)$, intensity $(I)$, which are intimately related to the way in which human beings perceive color. Second, the geometric distance between two colors does not mean the visual differences between the two colors in the RGB color space, which means using perceptual color space is more suitable for human intuition than using the primary colors (RGB). The cut image was mapped into the HSI color space, as shown in Figure 2c.

The most important step of image processing is image segmentation. It is the process of assigning a label to each pixel in an image so that pixels with the same label share certain features, such as color, 
intensity, or texture. There are various methods to implement segmentation, for example, thresholding methods, clustering methods, texture methods, transform methods, and so on. The image based on HSI color space was segmented with a thresholding technique, which replaced pixels in the image with a white pixel if the image intensity was greater than the threshold, or a black pixel if the image intensity was less than the threshold. The results of the repeated experiments showed that it was possible to segment accurately when choosing $H=200, S=50, I=200$ as the threshold values. The result is shown in Figure 2d. In order to eliminate the spatters, we first began with the open operation, then used the close operation for further processing, and the final result was shown in Figure 2e. As can be seen, the morphologic features of the metal vapor plume were well preserved.

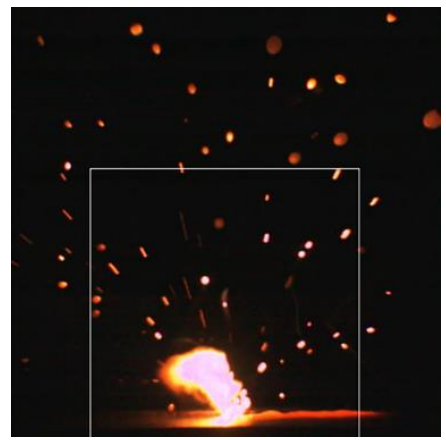

(a)

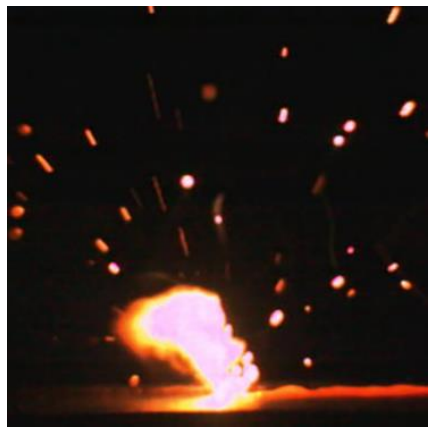

(b)

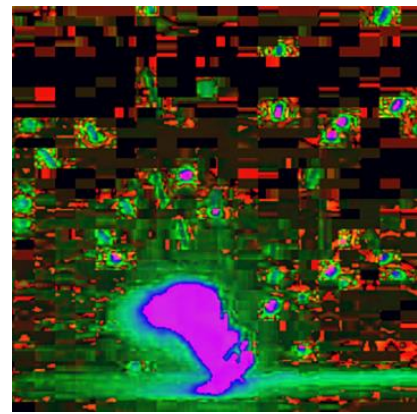

(c)

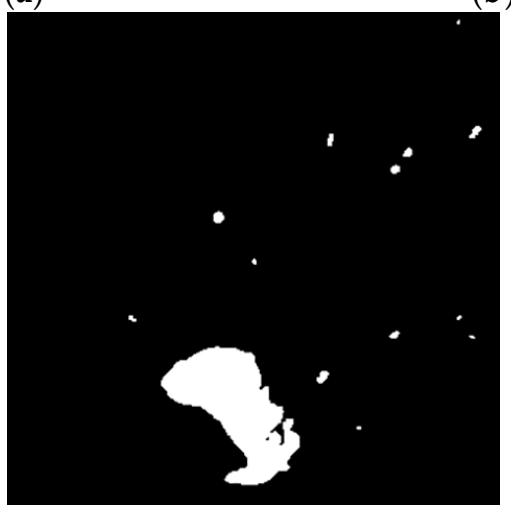

(d)

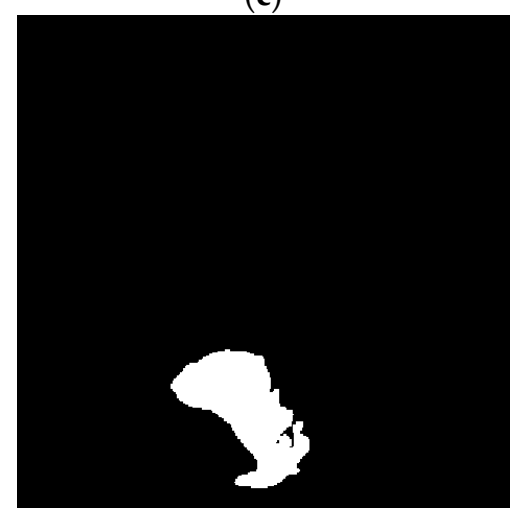

(e)

Figure 2. Schematic diagrams of color image processing: (a) the original RGB (red, green, blue) image; (b) the image has been cut by the white line box in Figure 2a; (c) the image has been converted into a HSI (hue, saturation, intensity) image; (d) image thresholding has been applied; (e) the spatters have been removed.

\subsection{Definition of Features}

After the color image was segmented, six features, which reflected the welding quality, were extracted. They were defined as the area, the height and the average intensity of metal vapor plume region, the horizontal coordinate and the vertical coordinate of plume centroid, and the number of spatters.

The pixel number of the effective plume was set equal to the area of the metal vapor plume, which was defined as:

$$
S=\sum_{j=1}^{n} \sum_{i=1}^{m} \operatorname{Binary}(i, j)
$$

where Binary $(i, j)$ was the value of pixel $(i, j)$.

The height of the metal vapor plume was defined as the vertical distance between the highest point and the lowest point. 
The average intensity of the metal vapor plume region was defined as:

$$
\bar{I}=\frac{\sum_{j=1}^{n} \sum_{i=1}^{m} I(i, j)}{n \times m}
$$

where $I(i, j)$ was the intensity of pixel $(i, j)$.

The centroid coordinate of the metal vapor plume region was defined as:

$$
x_{c}=\frac{\sum_{j=1}^{n} \sum_{i=1}^{m} g(i, j) \times i}{\sum_{j=1}^{n} \sum_{i=1}^{m} g(i, j)}, y_{c}=\frac{\sum_{j=1}^{n} \sum_{i=1}^{m} g(i, j) \times j}{\sum_{j=1}^{n} \sum_{i=1}^{m} g(i, j)}
$$

where $g(i, j)$ was the grey value of pixel $(i, j)$.

After the color image was segmented, we removed the region that had an area that was more than 450 pixels. The number of pixels in the remaining region was set equal to the number of spatters.

\subsection{SVM Method}

Support vector machine is a widely used classification model. The basic idea is to obtain the maximum-margin separating hyperplane in feature space $[21,26]$. For the training data set $T=\left\{\left(x_{1}, y_{1}\right),\left(x_{2}, y_{2}\right), \ldots\left(x_{n}, y_{n}\right)\right\}$, where $x_{i} \in R^{n}, y_{i} \in\{-1,1\}, i=1,2, \ldots, n$ and the selected penalty parameter $C>0$, Lagrange multipliers $\alpha=\left(\alpha_{1}, \alpha_{2}, \ldots, \alpha_{n}\right)$ were applied to construct a convex quadratic programming problem [26]:

$$
\begin{array}{ll}
\min _{\alpha} & \frac{1}{2} \sum_{i=1}^{n} \sum_{j=1}^{n} \alpha_{i} \alpha_{j} y_{i} y_{j}\left(x_{i} \cdot y_{j}\right)-\sum_{i=1}^{n} \alpha_{i} \\
\text { s.t. } & \sum_{i=1}^{n} \alpha_{i} y_{i}=0 \\
& 0 \leq \alpha_{i} \leq C, i=1,2, \ldots, n
\end{array}
$$

The solution $\alpha^{*}$ can be used to calculate $w^{*}$ and $b^{*}$, which are the normal vector and intercept of the separating hyperplane equation:

$$
w^{*} \cdot x+b^{*}=0
$$

Thus, the classification decision function is:

$$
f(x)=\operatorname{sign}\left(w^{*} \cdot x+b^{*}\right)
$$

When the classification problem is nonlinear, the kernel trick can be used, implicitly mapping the inputs into high-dimensional feature spaces. SVM can efficiently perform a nonlinear classification under the condition. The most widely used kernel function is the radial basis kernel function, which is shown as:

$$
K(x, z)=\exp \left(-\frac{\|x-z\|^{2}}{2 \sigma^{2}}\right)
$$

where $x$ and $z$ could be any sample, and $\sigma$ is a width parameter to control the radial range of the function.

\section{Result and Discussion}

\subsection{Features Extraction and Data Preprocessing}

During the whole welding process, 2400 images of the welding speed $4.5 \mathrm{~m} / \mathrm{min}$ were captured by the high-speed camera, and 1480 continuous images from No. 521 to 2000 were analyzed. The features, 
including the area, the height, the average intensity and the centroid coordinate of metal vapor plume region, and the number of spatters, were calculated. In order to eliminate the periodic fluctuation of the data and the influence of individual singularity, the extracted features were median filtered, as shown in Figure 3a-f.

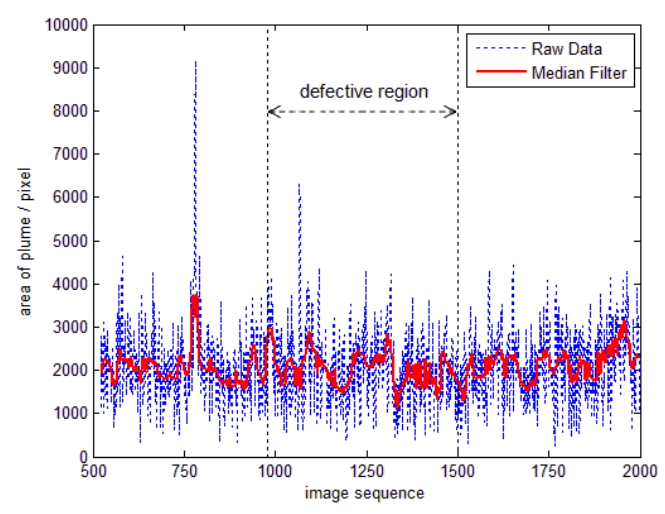

(a)

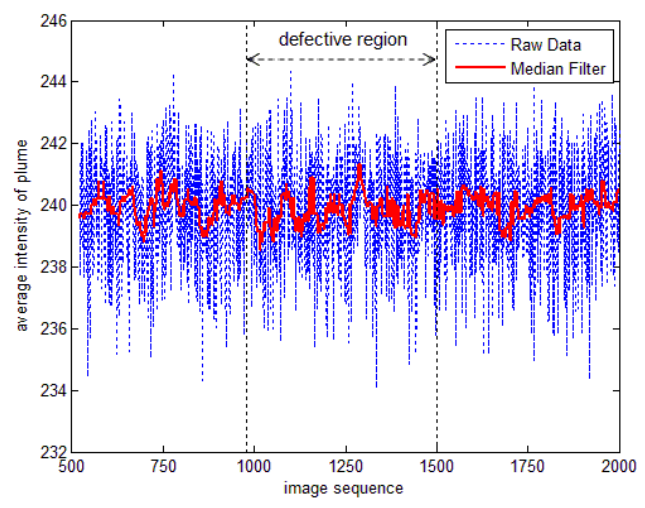

(c)

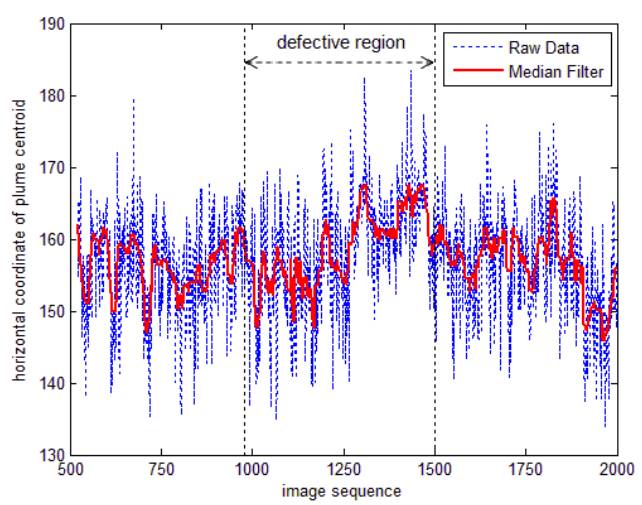

(e)

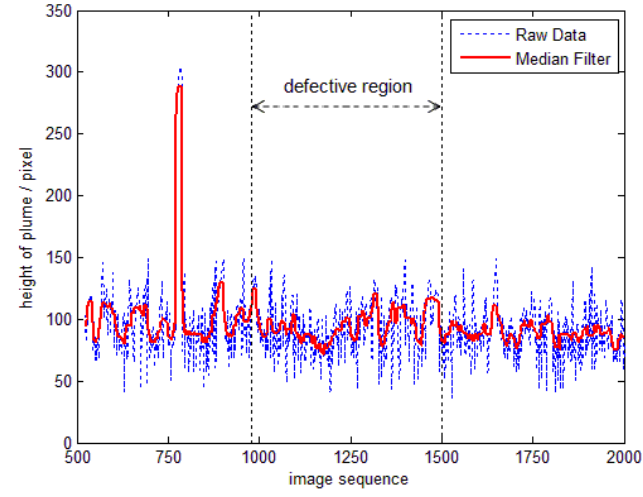

(b)

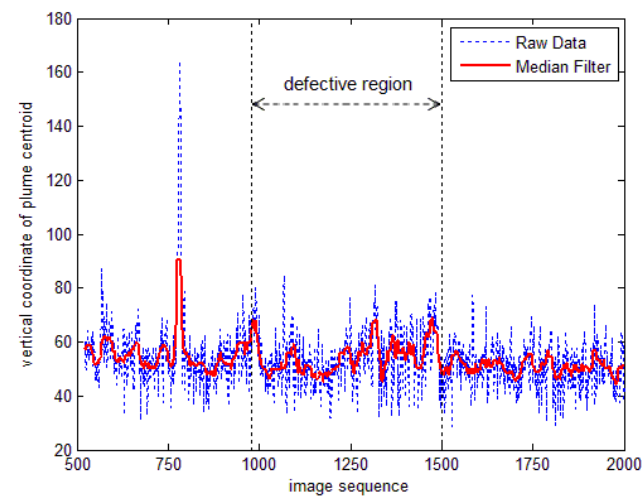

(d)

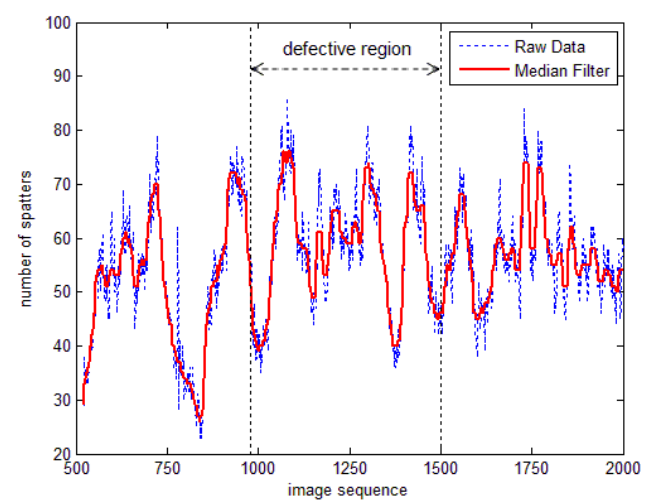

$(\mathbf{f})$

Figure 3. The extracted features: (a) the area of metal vapor plume; (b) the height of metal vapor plume; (c) the average intensity of metal vapor plume; (d) the vertical coordinate of plume centroid; (e) the horizontal coordinate of plume centroid; (f) the number of spatters.

\subsection{Features of the Welding Sample}

Figure 4 shows the top view of the welded seam. The welded seam was divided into three regions according to the image serial number, region A [521, 980], region B [981, 1500], and region C [1501, 2000], corresponding to the relevant region in Figure 4. Welding quality is mainly related to several factors, 
including the fluctuation in the welding process, the uniformity of the bead width of the weld, the number of internal pores, as well as the number and the size of spatters. From the surface appearance of the welded seam, the surface weld width of region $A$ and region $C$ were relatively stable and of high welding quality, while the weld width of region $B$ became narrow suddenly and was of poor weld quality. As such, region $A$ and region $C$ were treated as a class, denoted with $R$, and region $\mathrm{B}$ was treated as another class, denoted with $\mathrm{E}$.

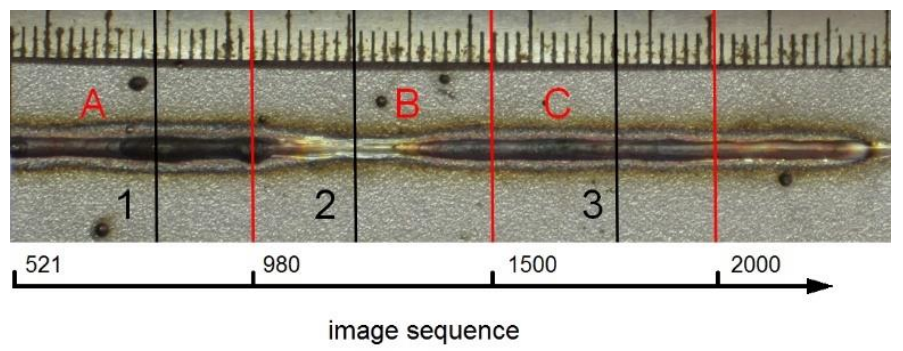

Figure 4. Top view of the welded seam. Three regions A, B, C were divided, and three different cross sections marked with 1,2 , and 3 were selected.

As shown in Figure 4, three different cross sections of the sample marked with 1,2, and 3 were selected for further observation of the internal quality. The bead width (the width of the welded seam in the horizontal direction on the sample surface) and the weld penetration of the three cross sections denoted 1, 2 and 3 were shown in Figure 5a-c. As shown in Figure 5 and Table 2, the depth-width ratio of the second cross sections was the largest, and the quality of its underfilled surface was the worst.

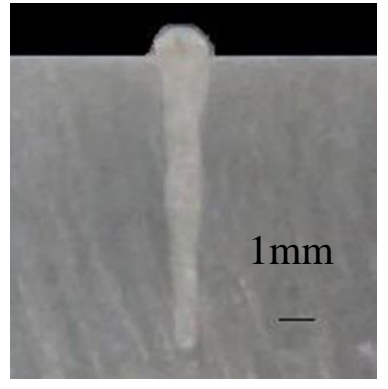

(a)

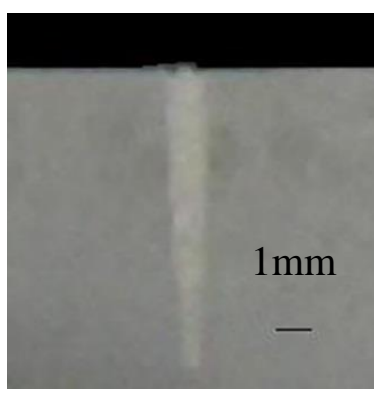

(b)

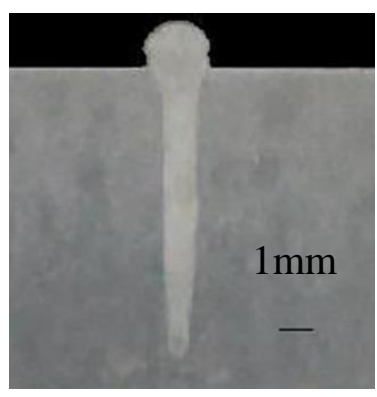

(c)

Figure 5. The traditional cross sections of the welded seam: (a) corresponding to region A; (b) corresponding to region $\mathrm{B}$; (c) corresponding to region $\mathrm{C}$.

Table 2. The depth and the depth-to-width ratio of the three traditional cross sections.

\begin{tabular}{cccc}
\hline Region & $\mathbf{1}$ & $\mathbf{2}$ & $\mathbf{3}$ \\
\hline The depth $(\mathrm{mm})$ & 8.05 & 8.3 & 8.4 \\
The width $(\mathrm{mm})$ & 1.66 & 1.43 & 1.66 \\
The depth-to-width ratio & 4.85 & 5.80 & 5.06 \\
\hline
\end{tabular}

Observing the cross sections through a metallurgical microscope (XJP-6/6A), several pores were found in the cross section, as shown in Figure 6. The captions of each image were named as $i-j$, which meant the $j$-th pores in the $i$-th cross sections. Two pores were found in the first cross section, as shown in Figure 6(1-1),(1-2), five pores were found in the second cross section, as shown in Figure 6(2-1)-(2-5), and two pores were found in the third cross section, as shown in Figure 6(3-1),(3-2). Most of the pores were rounded. The number of pores found in the second cross section were more than the other two sections, and the diameter of the pores were bigger than those of the other two sections. 
The first cross section belonged to region A, whose surface width was a bit wider. As can be seen from Figure 3, from the 771st to the 790th image, it was found that the area and the height of the metal vapor plume both underwent a sharp upward mutation. There existed two obvious raised knots on the surface of the welded seam in region A, which are some tumor-like particles caused by spatters, but only two pores were found in the corresponding cross section, which indicated that the sudden increase of the area and height of the metal vapor plume corresponded to the formation of the knots on the surface, but it had little relation with the change of the pores. We observed the trail of the spatters from the real-time video, and thought that the formation of knots was due to two reasons. First, when the spatters were big enough, the movement trail of spatters was mainly in the vertical direction under the action of its gravity, leading to the sudden increase of the area and height of metal vapor plume. Some spatters eventually fell onto the surface along the weld axis. The other reason is that knots were possibly from violent turbulence in the molten pool during welding.

The second cross section belonged to region B, whose surface width was the narrowest. By calculating the data in Figure 3, the average area and height of the metal vapor plume in region B were smaller than the other two regions, and five pores were found in the corresponding cross section. This indicated that the temperature of the molten pool in section B is relatively low, and that the solidification of liquid metal became faster. When the bubbles grew to a certain size and began to float, they were more likely to stay in the solidified weld metal to form pores if the floating speed was less than that of the crystallization of the metal pool.

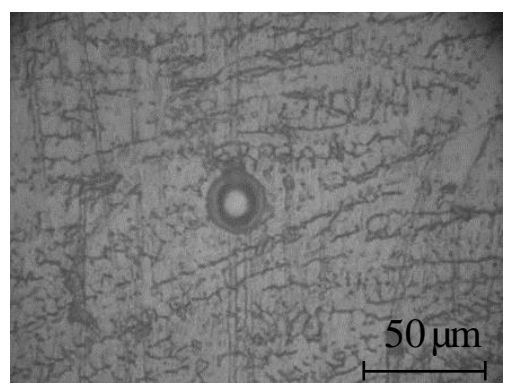

(1-1)

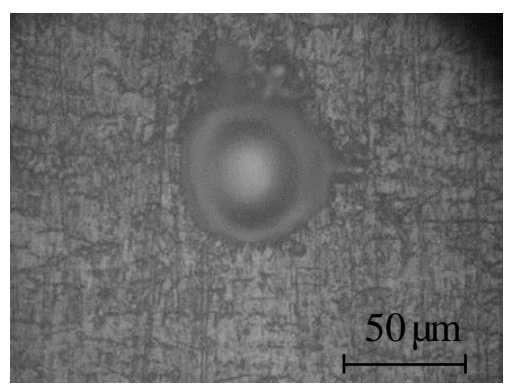

(2-2)

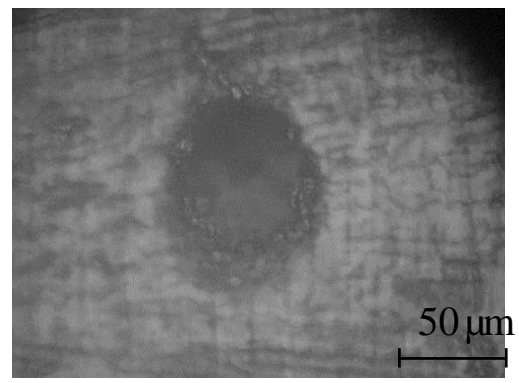

(2-5)

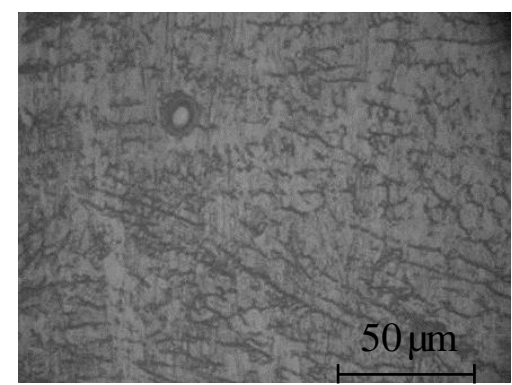

(1-2)

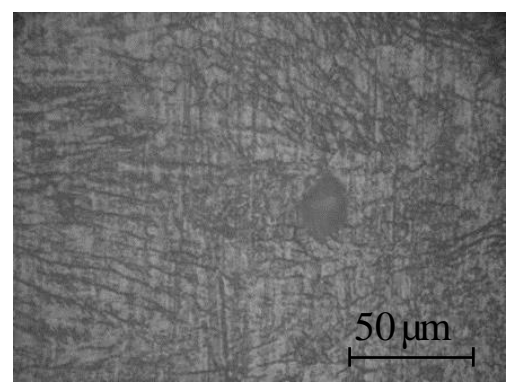

(2-3)

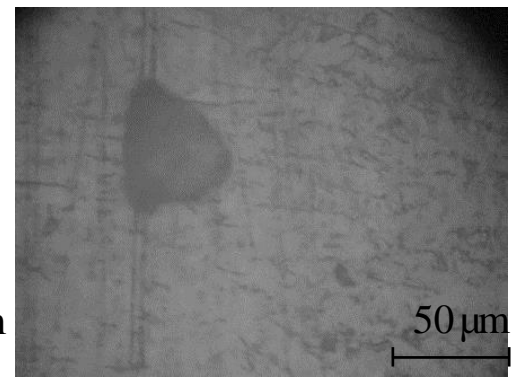

(3-1)

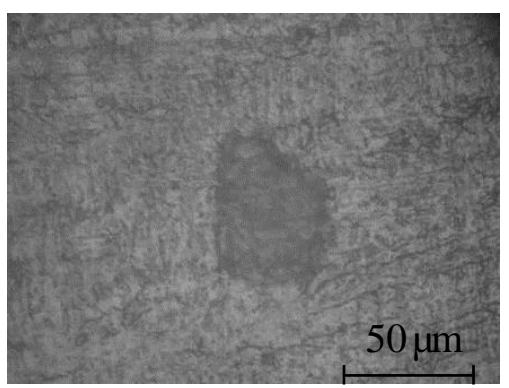

(2-1)

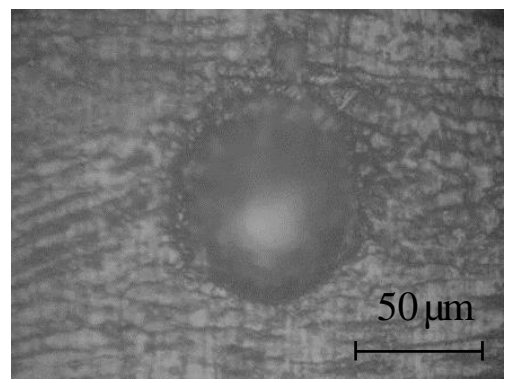

(2-4)

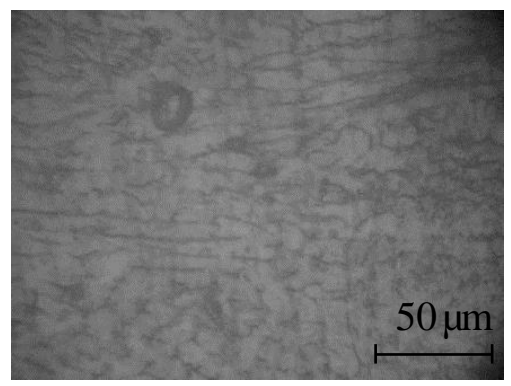

(3-2)

Figure 6. The pores in the cross section of the welded seam. The captions of each image were named as $i-j$, which meant the $j$-th pores in the $i$-th cross sections. 
3.3. Classification Experiment Based on the SVM Approach, Quadratic Discriminant Analysis, and Linear Discriminant Analysis

After median filtering, support vector machine, linear discriminant analysis [27], and quadratic discriminant analysis [28] were used to build models using each feature separately. In practice, 10-fold cross-validation is widely used to test the accuracy of algorithms [17,29], which meant the entire data set was divided into 10 equal parts, nine parts were taken as training sets and the remaining one was used as a test set. Experiments were conducted ten times, and the average result was taken as the accuracy rate of the model. The results are shown in Table 3 and Figure 7, where the features were sorted in descending order according to the accuracy rate of SVM.

Table 3. The classification results of SVM, quadratic discriminant analysis, and linear discriminant analysis.

\begin{tabular}{|c|c|c|c|c|}
\hline No. & Feature & SVM (\%) & Quadratic (\%) & Linear (\%) \\
\hline 1 & area of plume & 83.04 & 64.86 & 64.86 \\
\hline 2 & height of plume & 72.77 & 52.97 & 64.86 \\
\hline 3 & $\begin{array}{l}\text { horizontal coordinate of } \\
\text { plume centroid }\end{array}$ & 72.43 & 72.36 & 71.96 \\
\hline 4 & number of spatters & 71.62 & 65.81 & 66.28 \\
\hline 5 & $\begin{array}{l}\text { vertical coordinate of } \\
\text { plume centroid }\end{array}$ & 68.72 & 64.46 & 63.85 \\
\hline 6 & average intensity of plume & 64.93 & 66.42 & 65.27 \\
\hline
\end{tabular}

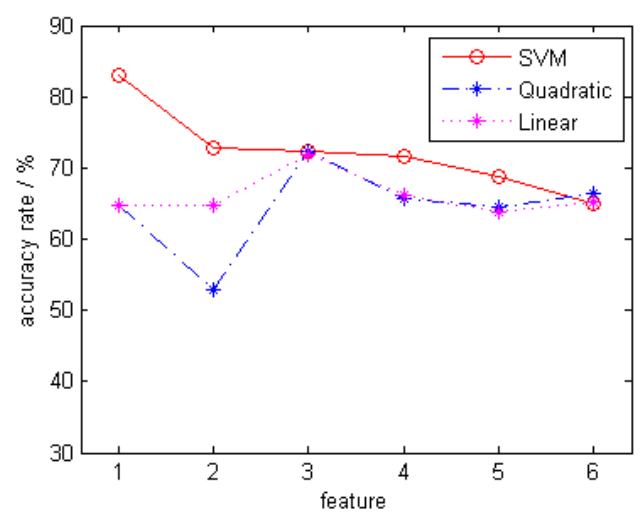

Figure 7. The classification results of various methods.

As shown in Table 3, the accuracy rate of SVM was significantly higher than that of quadratic discriminant analysis and linear discriminant analysis when using Feature 1, 2, 4 and 5. For Feature 3 and Feature 6, the accuracy rate of SVM was a little better and worse than the other two models, respectively. In other words, the results showed that SVM was more suitable for predicting the welding quality in the experiment. There were two possible reasons. On one hand, SVM was based on margin maximization, which ensured its advantage in generalization ability. On the other hand, as the kernel function was used, SVM could analyze and classify data not only in feature space, but also in high-dimensional space.

The experimental results above showed that the accuracy rate could reach $83.04 \%$ when using SVM with Feature 1. To further improve the classification ability, two other features were chosen based upon Pearson product-moment correlation coefficient. The Pearson product-moment correlation coefficient $r$ between two features $x$ and $y$ was calculated by [30]: 


$$
\begin{gathered}
r=\frac{\sum_{i=1}^{n}\left(a_{i}-\bar{a}\right)\left(b_{i}-\bar{b}\right)}{\sqrt{\sum_{i=1}^{n}\left(a_{i}-\bar{a}\right)^{2} \cdot \sum_{i=1}^{n}\left(b_{i}-\bar{b}\right)^{2}}} \\
\bar{a}=\frac{\sum_{i=1}^{n} a_{i}}{n}, \bar{b}=\frac{\sum_{i=1}^{n} b_{i}}{n}
\end{gathered}
$$

where $n$ was the size of sample, and $r$ was a measure of the correlation between two features. A greater absolute value of $r$ indicated stronger correlation.

Pearson correlation coefficients between each feature and Feature 1 are shown in Table 4 . The features of the table were sorted by the absolute value of $r$ in ascending order. The absolute value of the correlation coefficient between Feature 4 and Feature 1 was about 0.0665 , which indicated that the correlation between them was very weak and thus adding Feature 4 to the subset would provide lots of new information. The second lowest correlation coefficient was between Feature 3 and Feature 1, and the value was close to 0.2210 , which meant the new information it could provide was still considerable. The coefficient $r$ between other features and Feature 1, such as $0.4497,0.5225$ and 0.5603 , indicated that they had much stronger correlations with Feature 1 , and therefore adding them to the subset should not be able to improve the classification accuracy as much as Feature 4 and Feature 3.

Table 4. Pearson correlation coefficients $r$ between each feature and Feature 1.

\begin{tabular}{cccc}
\hline No. & Feature & $|\boldsymbol{r}|$ & $\boldsymbol{r}$ \\
\hline 4 & number of spatters & 0.0665 & -0.0665 \\
3 & horizontal coordinate of plume centroid & 0.2210 & -0.2210 \\
6 & average intensity of plume & 0.4497 & 0.4497 \\
2 & height of plume & 0.5225 & -0.5225 \\
5 & vertical coordinate of plume centroid & 0.5603 & -0.5603 \\
1 & area of plume & 1.0000 & 1.0000 \\
\hline
\end{tabular}

Scatter plots of three selected features are shown in Figure 8. As can be observed in the figure, most of the data from different classes were distributed in different areas, which could not be easily separated by linear or quadratic models. More complex and nonlinear models, such as SVM, were needed.

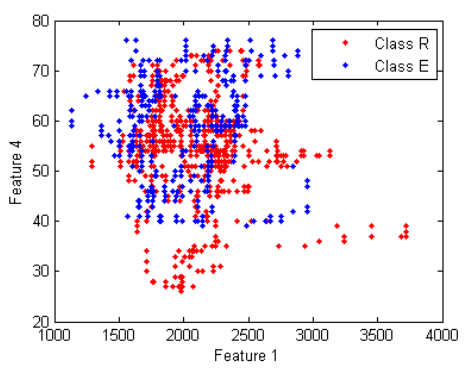

(a)

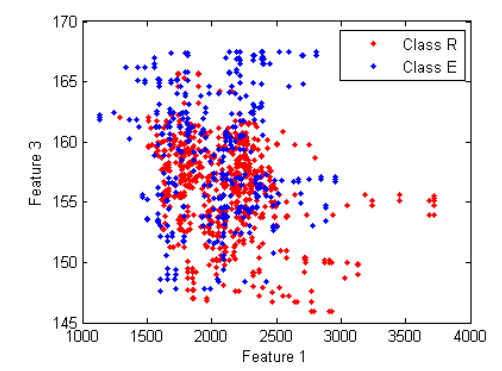

(b)

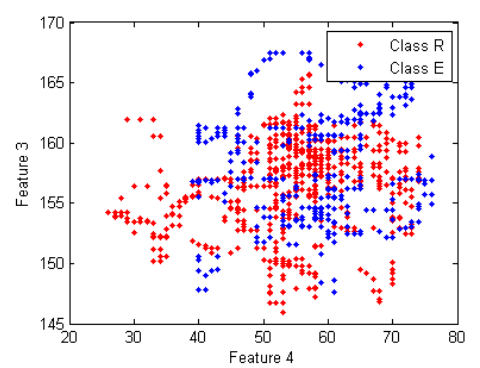

(c)

Figure 8. Scatter plots of selected features: (a) Feature 4 vs. Feature 1; (b) Feature 3 vs. Feature 1; (c) Feature 3 vs. Feature 4.

The classification results using the Feature 1, Feature 4, and Feature 3 above are shown in Table 5, and the confusion matrices for each experiment are shown in Tables 6-8. 
Table 5. The classification result using the three features.

\begin{tabular}{cccccc}
\hline ID & Model & Feature(s) & \multicolumn{2}{c}{ Accuracy Rate (\%) } & \multirow{2}{*}{$\begin{array}{c}\text { Overall Accuracy } \\
\text { Rate (\%) }\end{array}$} \\
\cline { 3 - 4 } & & Class E & Class R & \\
\hline 1 & SVM & 1 & 68.65 & 90.83 & 83.04 \\
2 & SVM & 1,4 & 81.92 & 96.77 & 91.55 \\
3 & SVM & $1,4,3$ & 82.69 & 99.48 & 93.58 \\
\hline
\end{tabular}

Table 6. Confusion matrices for cross-validation.

\begin{tabular}{cccc}
\hline \multirow{2}{*}{ SVM (1) } & \multicolumn{2}{c}{ Predicted Class } \\
\cline { 3 - 4 } & & Class E & Class R \\
\hline \multirow{2}{*}{ Actual Class } & Class E & $68.65 \%(357 / 520)$ & $31.35 \%(163 / 520)$ \\
& Class R & $9.17 \%(88 / 960)$ & $90.83 \%(872 / 960)$ \\
\hline
\end{tabular}

Table 7. Confusion matrices for cross-validation.

\begin{tabular}{cccc}
\hline \multirow{2}{*}{ SVM (1,4) } & \multicolumn{2}{c}{ Predicted Class } \\
\cline { 3 - 4 } & & Class E & Class R \\
\hline \multirow{2}{*}{ Actual Class } & Class E & $81.92 \%(426 / 520)$ & $18.08 \%(94 / 520)$ \\
& Class R & $3.23 \%(31 / 960)$ & $96.77 \%(929 / 960)$ \\
\hline
\end{tabular}

Table 8. Confusion matrices for cross-validation.

\begin{tabular}{cccc}
\hline \multirow{2}{*}{ SVM (1,4,3) } & \multicolumn{2}{c}{ Predicted Class } \\
\cline { 3 - 4 } & & Class E & Class R \\
\hline \multirow{2}{*}{ Actual Class } & Class E & $82.69 \%(430 / 520)$ & $17.31 \%(90 / 520)$ \\
& Class R & $0.52 \%(5 / 960)$ & $99.48 \%(955 / 960)$ \\
\hline
\end{tabular}

From Tables 5-8, it was noticed that the performance of SVM was improved with the introduction of features. Using only Feature 1, the accuracy rate was only $83.04 \%$. As Feature 4 was added to the feature subset, the accuracy rate increased to $91.55 \%$, especially the accuracy rate of Class E had a sharp increase from $68.65 \%$ to $81.92 \%$. With all three selected features, the accuracy rate reached $93.58 \%$. Consequently, SVM was able to achieve satisfactory classification performance with new information provided by the two additional features

\section{Conclusions}

(1) During the high-power laser welding process, high-speed photography was used to obtain dynamic metal vapor plume images. Six features were extracted and defined. The experimental results show that three features, including the area of plume, number of spatter, and horizontal coordinate of plume centroid, are closely related to the welding quality. A SVM model based on these three features was established and $93.58 \%$ of 10 -fold cross-validation accuracy rate was achieved, which indicates high prediction and generalization ability. As such, it is feasible to predict the welding performance and be applied in the control welding process.

(2) The combination of SVM and Pearson product-moment correlation coefficient was used to select the features with the best predictive capacity. The method is simple and effective, which provides a valuable technique to select features for characterizing the welding quality in the welding process.

A real-time monitoring model of welding status was established based on the consideration of laser-induced metal vapor plume by combining modeling with experimental testing, which helped 
ensure high reliability, and then on the basis of the results, a welding quality prediction model was established based on the characteristics of the metal vapor plume, so that it can be applied to real-time monitoring in the high-power laser welding process.

Acknowledgments: This work was financially supported by the National Natural Science Foundation of China (51505158, 11674106), and the Natural Science Foundation of Guangdong Province (2014A030310153). Many thanks are given to Katayama Laboratory of Joining and Welding Research Institute of Osaka University for the technical assistance of laser welding experiments.

Author Contributions: Teng Wang and Xiangdong Gao conceived and designed the experiments; Xiangdong Gao performed the experiments; Teng Wang, Juequan Chen, and Wei Li analyzed the data and wrote the paper.

Conflicts of Interest: The authors declare that there is no conflict of interest regarding the publication of this paper.

\section{References}

1. Nilsen, M.; Sikström, F. Monitoring of laser beam welding by a non-intrusive optical sensor system using photodiodes. In Proceedings of the JOM 18 International Conference on Joining Materials, Helsingör, Danmark, 26-29 April 2015; pp. 1-14.

2. Stritt, P.; Boley, M.; Heider, A.; Fetzer, F.; Jarwitz, M.; Weller, D.; Weber, R.; Berger, P.; Graf, T. Comprehensive process monitoring for laser welding process optimization. In Proceedings of SPIE Conference 9741, High-Power Laser Materials Processing: Lasers, Beam Delivery, Diagnostics, and Applications V, San Francisco, CA, USA, 13-18 March 2016; pp. 97410Q:1-97410Q:10.

3. Sibillano, T.; Ancona, A.; Rizzi, D.; Lupo, V.; Tricarico, L.; Lugarà, P.M. Plasma Plume Oscillations Monitoring during Laser Welding of Stainless Steel by Discrete Wavelet Transform Application. Sensors 2010, 10, 3549-3561. [CrossRef] [PubMed]

4. Atabaki, M.M.; Yazdian, N.; Ma, J.; Kovacevic, R. High power laser welding of thick steel plates in a horizontal butt joint configuration. Opt. Laser Technol. 2016, 83, 1-12. [CrossRef]

5. Bebiano, D.; Alfaro, S.C.A. A Weld Defects Detection System Based on a Spectrometer. Sensors 2009, 9, 2851-2861. [CrossRef] [PubMed]

6. Ogawa, Y. Various Measurement technology of welding process using a high-speed video camera. J. Jpn. Weld. Soc. 2015, 84, 25-30. [CrossRef]

7. Gao, X.D.; Zhang, Y.X. Prediction model of weld width during high-power disk laser welding of 304 austenitic stainless steel. Int. J. Precis. Eng. Manuf. 2014, 15, 399-405. [CrossRef]

8. Huang, W.; Kovacevic, R. A Laser-Based Vision System for Weld Quality Inspection. Sensors 2011, 11, 506-521. [CrossRef] [PubMed]

9. Alfaro, S.C.A.; Cayo, E.H. Sensoring Fusion Data from the Optic and Acoustic Emissions of Electric Arcs in the GMAW-S Process for Welding Quality Assessment. Sensors 2012, 12, 6953-6966. [CrossRef] [PubMed]

10. Luo, Y.; Du, Y.; Zhu, L.; Han, J.T.; Wan, R.; Xie, X.J. Study on the thermo-effect of P-GMAW characterized by structure-borne acoustic emission signals detected in welding on aluminum alloy. Measure 2016, 92, 200-207. [CrossRef]

11. Li, Z.Y.; Wang, W.; Wang, X.Y.; Li, H. A study of the radiation of a Nd:YAG laser-MIG hybrid plasma. Opt. Laser Technol. 2010, 42, 132-140. [CrossRef]

12. Miyagi, M.; Zhang, X. Investigation of laser welding phenomena of pure copper by $x$-ray observation system. J. Laser Appl. 2015, 27, 33-34. [CrossRef]

13. Pinto-Lopera, J.E.; Mauricio, S.T.; Motta, J.M.; Absi Alfaro, S.C. Real-Time Measurement of Width and Height of Weld Beads in GMAW Processes. Sensors 2016, 16, 1500. [CrossRef] [PubMed]

14. Li, S.C.; Chen, G.Y.; Katayama, S.; Zhang, Y. Relationship between spatter formation and dynamic molten pool during high-power deep-penetration laser welding. Appl. Surf. Sci. 2014, 301, 481-488. [CrossRef]

15. You, D.Y.; Gao, X.D.; Katayama, S.J. Multisensor Fusion System for Monitoring High-Power Disk Laser Welding Using Support Vector Machine. IEEE Trans. Ind. Inform. 2014, 10, 1285-1295.

16. $\mathrm{He}, \mathrm{K}$; $\mathrm{Li}, \mathrm{X}$. A quantitative estimation technique for welding quality using local mean decomposition and support vector machine. J. Intell. Manuf. 2014, 25, 1-9. [CrossRef]

17. Shao, C.H.; Paynabar, K.; Kim, T.H.; Jin, J.J.; Hu, S.J.; Spicer, J.P.; Wang, H.; Abell, J.A. Feature selection for manufacturing process monitoring using cross-validation. J. Manuf. Syst. 2013, 32, 550-555. [CrossRef] 
18. Shao, C.H.; Kim, T.H.; Hu, S.J.; Jin, J.J.; Abell, J.A.; Spicer, J.P. Tool wear monitoring for ultrasonic metal welding of lithium-ion batteries. J. Manuf. Sci. Eng. 2016, 138, 051005. [CrossRef]

19. Wu, Z.; Shamsuzzaman, M. Design and application of integrated control charts for monitoring process mean and variance. J. Manuf. Syst. 2005, 24, 302-314. [CrossRef]

20. Suriano, S.; Wang, H.; Hu, S.J. Sequential monitoring of surface spatial variation in automotive machining processes based on high definition metrology. J. Manuf. Syst. 2012, 31, 8-14. [CrossRef]

21. Alpaydin, E. Introduction to Machine Learning, 2nd revised ed.; MIT Press: Cambridge, MA, USA, 2010.

22. Wang, X.; Qin, B.; Xu, H.; Zhu, W. Rotary drying process modeling and online compensation. Control Eng. Pract. 2015, 41, 38-46. [CrossRef]

23. Mekhalfa, F.; Nacereddine, N. Multiclass Classification of Weld Defects in Radiographic Images Based on Support Vector Machines. In Proceedings of the 10th International Conference on Signal-Image Technology and Internet-Based Systems, Marrakech, Morroco, 23-27 November 2014; pp. 1-6.

24. Chen, B.; Zhang, H.T.; Feng, J.C.; Chen, S.B. A study of welding process modeling based on Support Vector Machines. In Proceedings of the International Conference on Computer Science and Network Technology, Harbin, China, 24-26 December 2011; pp. 1859-1862.

25. Gao, L.W.; Xue, J.X.; Lin, F. Intelligentized evaluation on dynamic characteristics of $\mathrm{CO}_{2}$ arc welding power source based on key Eigen values and support vector machine. In Proceedings of the 4th International Congress on Image and Signal Processing, Shanghai, China, 15-17 October 2011; pp. 2545-2548.

26. Cristianini, N. An Introduction to Support Vector Machines; Machine Press: Beijing, China, 2005.

27. Fisher, R.A. The Use of Multiple Measurements in Taxonomic Problems. Ann. Eugen. 1936, 7, 179-188. [CrossRef]

28. Zhang, M.Q. Identification of protein coding regions in the human genome by quadratic discriminant analysis. Proc. Natl. Acad. Sci. USA 1997, 94, 565-568. [CrossRef] [PubMed]

29. Mery, D. Automated detection of welding discontinuities without segmentation. Mater. Eval. 2011, 69, 656-663.

30. Rodgers, J.L.; Nicewander, W.A. Thirteen ways to look at the correlation coefficient. Am. Stat. 1988, 42, 59-66. [CrossRef]

(C) 2017 by the authors. Licensee MDPI, Basel, Switzerland. This article is an open access article distributed under the terms and conditions of the Creative Commons Attribution (CC BY) license (http:/ / creativecommons.org/licenses/by/4.0/). 